\title{
Numerical Analysis of the Effect of Mesoscale Eddies on Seismic Imaging
}

\author{
LILI $\mathrm{J}_{\mathrm{I}}{ }^{1}$ and MIAN LIN ${ }^{1}$
}

\begin{abstract}
The influence of the mesoscale eddy on seismic wave propagation and seismic imaging in deep sea is investigated. Based on fundamental fluid equations, an appropriate partial differential equation is derived for the acoustic pressure field in water with eddies, including current effects. Seismic wavefields and synthetic seismograms in the center of the eddy are simulated. Numerical experiments demonstrate that velocity variations caused by the eddy can lead to traveltime perturbations. Further, in seismic images, the reflectors below the water layer are positioned incorrectly due to the perturbation of the eddy and this image perturbation depends linearly on the migration velocity of the layer below the corresponding reflector. The zero-offset seismic profiling throughout the affected area of the eddy shows that the maximum traveltime perturbation appears at the center of the eddy and the structure of horizontal reflectors below the water layer are distorted.
\end{abstract}

Key words: Partial differential equation, synthetic seismogram, seismic imaging, depth perturbation.

\section{Introduction}

Exploration and development of offshore hydrocarbon resources has advanced into remote deepwater regions over the last decade (Hough et al., 2011; VARTANYAN, 2010). Development drilling targets increasingly requires precise in both horizontal and vertical positioning. Production facility design errors, either over-building or under-building, can yield heavy losses in complicated and costly industrial activity. However, the development of deepwater field continues to have imaging problems which is due in large measure to complex conditions in deepwater. Recently, the studies in seismic oceanography have shown that the mesoscale eddies in deep sea can be

1 Laboratory of Environment Mechanics, Institute of Mechanics, Chinese Academy of Sciences, Beisihuan Road 15, Haidian District, Beijing 100190, China.

E-mail: jilili@imech.ac.cn; linmian@imech.ac.cn imaged through processing the seismic reflection data (BIESCAS et al., 2008; Song et al., 2008, 2009; Hu et al., 2009; PinHeiro et al., 2010). Some geophysicists have found that the temperature steps are typically large enough, a few hundredths of a degree over only a few meters, to be seen as reflecting features in seismic data. Depending on the sign of the temperature change caused by eddies reflections may undergo a $180^{\circ}$ phase change or not (Gonella and Michon, 1988; HolbrooK et al., 2003; BeniteZ-Nelson and McGillicuddy, 2008; Krahmann et al., 2008; Wood et al., 2008). The results provide us with insight that the existence of the mesoscale eddies should have influenced seismic imaging. Thus in this paper, we investigate the effect of mesoscale eddy which are observed more frequently in the South China Sea.

Mesoscale eddies are large coherently rotating bodies of water and commonly appeared in deepwater. A striking environmental characteristic of eddies is a large distortion of the normally horizontal isotherms and eddies have been shown to be responsible for isothermal uplifts of 500 m or more (HENRICK et al., 1977, 1980; Nysen and Power, 1978; Baer, 1980). Much attention has been focused on mesoscale eddies by underwater acousticians and physical oceanographers other than seismologists. In underwater acoustics, the propagation of acoustic wave is treated with quasi-horizontal direction (large distance propagation), while seismic model deals with quasi-vertical propagation (LIU and TAO, 1997; JENSEn et al., 2000; KoRmann et al., 2010). Thus underwater acoustic propagation methods are not optimal to be applied to seismic models. Recently, KORMANN et al. simulated seismic oceanography experiments numerically by using a simplified wave equation and second-order complex frequency shifted perfectly matched layers (PML) at the numerical boundaries (Kormann et al., 2008, 2010; Kormann, 2009). This simplified wave equation is derived based on two 
basic assumptions: one is that the density $\rho$ and the sound speed $c$ of the fluid satisfied $c=\sqrt{K / \rho}$ where $K$ is the compression modulus of the fluid; the other is that the fluid is static or moving with uniform speed. However, for the analytic eddy model in our paper, the relation between the density and the sound speed of mesoscale eddies are much more complicated (HENRICK et al., 1977). Further, as mentioned above, in mesoscale eddies the water rotates with a nonuniform velocity, so a wave equation including nonuniform current effect is more desirable (ROBERTSON et al., 1985). In this paper, to investigate the effect of mesoscale eddy, an appropriate partial differential equation is derived for the acoustic pressure field while including the density variations and nonuniform currents in the eddy.

The outline of this paper is as follows: in Sect. 2, the derivation of the appropriate partial differential equation is briefly presented. Subsequently, the PML are introduced to avoid spurious reflections at the lateral boundaries. In Sect. 3, we examine the characteristics of wavefields and synthetic seismograms for having eddy and no-eddy case. Then we demonstrate that the image perturbation caused by the eddy is dependent on the arrival time perturbation and the migration velocity below water. Finally, the distortion of migrated images of the target stratum is discussed.

\section{Methodology}

Our physical model is depicted in Fig. 1. In this paper, the eddy is based on an analytic model that reproduces observed data in the South China Sea (Wu et al., 2001; JIAN et al., 2009). The effective depth of the eddy is $1.6 \mathrm{~km}$ and the effective radius is $150 \mathrm{~km}$ in horizontal range. The water depth is $2,200 \mathrm{~m}$, and below water there are two homogeneous layers separated by flat interfaces $H_{1}$ and $H_{2}$. It should be pointed out that the water is inhomogeneous in both horizontal and vertical direction.

\subsection{Equations for Inhomogeneous Moving Water}

The general governing equations (TEMKIN, 1981) for the motion of an adiabatic and nondissipative fluid are:

$$
\begin{gathered}
\frac{\partial \widehat{\rho}_{\mathrm{w}}}{\partial \hat{t}}+\nabla \cdot\left(\widehat{\rho}_{\mathrm{w}} \widehat{\mathbf{v}}_{\mathrm{w}}\right)=0 \\
\widehat{\rho}_{\mathrm{w}} \frac{D \widehat{\mathbf{v}}_{\mathrm{w}}}{D \widehat{t}}=-\nabla \widehat{P}_{\mathrm{w}} \\
\widehat{c}_{\mathrm{w}}^{2} \frac{D \widehat{\rho}_{\mathrm{w}}}{D \widehat{t}}=\frac{D \widehat{P}_{\mathrm{w}}}{D \widehat{t}}
\end{gathered}
$$

where $\nabla$ is the gradient operator, $D / D \widehat{t}=(\partial / \partial \widehat{t}+$ $\left.\widehat{\mathbf{v}}_{\mathrm{w}} \times \nabla\right), \hat{\rho}_{\mathrm{w}}$ is fluid density, $\widehat{\mathbf{v}}_{\mathrm{w}}$ is the fluid velocity vector, $\widehat{P}_{\mathrm{w}}$ is pressure in water, $\widehat{c}_{\mathrm{w}}$ is sound speed in water.

The quantities $\hat{\rho}_{\mathrm{w}}, \widehat{P}_{\mathrm{w}}, \widehat{\mathbf{v}}_{\mathrm{w}}$ in Eqs. (1a)-(1c) are assumed to have the following form (RoBERTSON et al., 1985):

$$
\widehat{p}_{\mathrm{w}}=\widehat{p}_{\mathrm{w} 0}+\widehat{p}_{\mathrm{w} 1}, \widehat{\rho}_{\mathrm{w}}=\widehat{\rho}_{\mathrm{w} 0}+\widehat{\rho}_{\mathrm{w} 1}, \widehat{\mathbf{v}}_{\mathrm{w}}=\widehat{\mathbf{v}}_{\mathrm{w} 0}+\widehat{\mathbf{v}}_{\mathrm{w} 1}
$$

The quantities are composed of two components. One describes the state in absence of an acoustic disturbance, indicated by zero subscript, and the other represents acoustic perturbation components caused by eddies, indicated by a unit subscript.

We assumed that the fluid density $\hat{\rho}_{\mathrm{w}}(\widehat{x}, \widehat{z}, \widehat{t})$ and the velocities $\widehat{c}_{\mathrm{w}}(\hat{x}, \hat{z})$ and $\widehat{\mathbf{v}}_{\mathrm{w}}(\hat{x}, \hat{z}, \widehat{t})$ of sound and fluid, respectively, depend arbitrarily on the vertical coordinate $\hat{z}$ and weakly on the horizontal coordinates $\hat{x}$.

To nondimensionalize Eqs. (1a)-(1c) and (2), we let:

$$
\begin{gathered}
\widehat{x}=x \widehat{L}, \widehat{z}=z \widehat{L}, \widehat{t}=t \widehat{T} \\
\widehat{\mathbf{v}}_{\mathrm{w} 0}(\widehat{z})=\widehat{U}_{0} \widehat{\mathbf{v}}_{0}(z)=\widehat{U}_{0} u_{0}(z) \mathbf{i} \\
\widehat{\mathbf{v}}_{\mathrm{w} 1}(\hat{x}, \widehat{z}, \widehat{t})=\delta \widehat{c}_{0} \mathbf{v}_{1}(x, z, t), \\
\widehat{\rho}_{\mathrm{w} 0}(\hat{x}, \widehat{z})=\widehat{\rho}_{00} \rho_{0}(x, z), \\
\widehat{\rho}_{\mathrm{w} 1}(\hat{x}, \widehat{z}, \widehat{t})=\delta \widehat{\rho}_{00} \rho_{1}(x, z, t), \\
\widehat{p}_{0}(\hat{x}, \widehat{z})=\widehat{\rho}_{00} \widehat{U}_{0}^{2} p_{0}(x, z) \\
\widehat{p}_{1}(\widehat{x}, \widehat{z}, \widehat{t})=\delta \widehat{\rho}_{00} \widehat{c}_{0} \widehat{L} \widehat{T}^{-1} p_{1}(x, z, t)
\end{gathered}
$$

where $\widehat{L}$ and $\widehat{T}$ are characteristic length and time scales to be specified later, $\hat{\rho}_{00}, \widehat{U}_{0}$ and $\widehat{c}_{0}$ are a reference density, current speed and sound speed, 

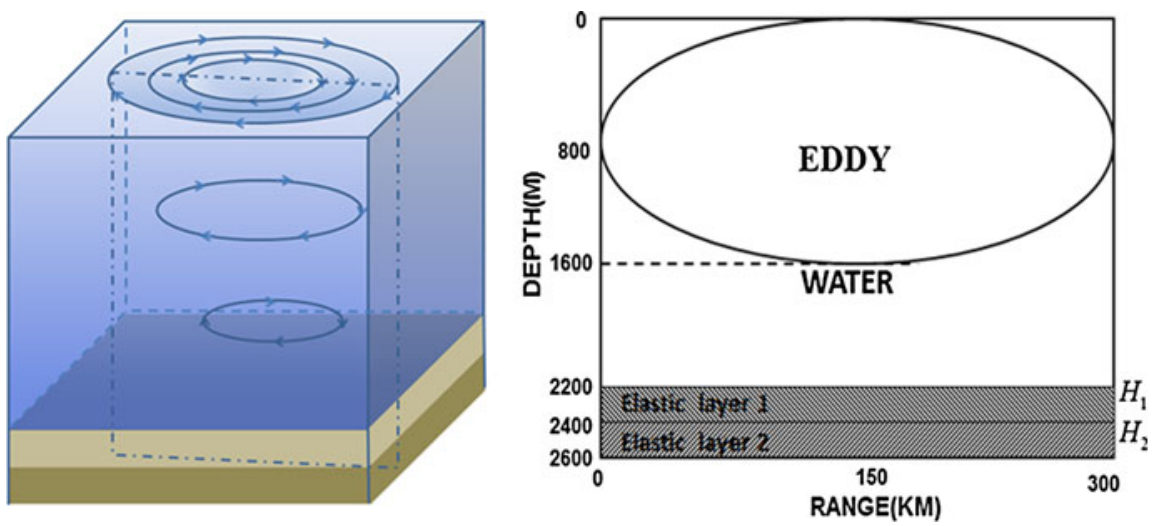

Figure 1

Physical Model

respectively. $u_{0}, \mathbf{v}_{1}, p_{0}, p_{1}, \rho_{0}$ and $\rho_{1}$ are dimensionless quantities of order of magnitude unity, $\mathbf{I}$ is the unit vector along the $x$ axis, and $\delta$ which represents the acoustic perturbation is a small dimensionless number.

Substituting Eqs. (2)-(4f) into Eqs. (1a)-(1c) and neglecting the terms of high order, we obtain (see Sect. "5")

$$
\begin{aligned}
\mu \rho_{0} \nabla\left(\frac{1}{\rho_{0}} \nabla p_{1 t}\right)= & 2 M \mu n^{2} u_{0} p_{1 x t t} \\
& -2\left[M /\left(\mu \rho_{0}^{2}\right)\right] u_{0}^{\prime} p_{1 z x}+\mu^{2} n^{2} p_{1 t t}
\end{aligned}
$$

where $\mu=\widehat{L} \widehat{c}_{0}^{-1} \widehat{T}^{-1}, M$ denotes the mach number $\widehat{U}_{0} / \widehat{c}_{0}$.

Next, we rescale the current gradient $u_{0}^{\prime}$ to indicate its actual magnitude. This is necessary because small currents may give rise to rather large current shears (ROBERTSON et al., 1985). Thus, the current gradient can be expressed as

$$
u_{0}^{\prime}=\xi G(z)
$$

where $G$ is an order unity function. Here we define $\xi$ is as the maximum of $\left|u_{0}^{\prime}\right|$ over all depth $z$. In order to indicate possible values for $\xi$, we consider that depth at which the maximum current shear occurs. In the vicinity of this depth, $G \cong 1$. In particular, our scaling can be used to express $u_{0}^{\prime}$ as

$$
u_{0}^{\prime}=\widehat{L} \widehat{U}_{0}^{-1} \frac{\mathrm{d} \widehat{u}_{0}}{\mathrm{~d} \widehat{z}}=\left(\frac{\widehat{c}_{0}}{2 \pi \widehat{f} \widehat{U}_{0}}\right)\left(\frac{\mathrm{d} \widehat{u}_{0}}{\mathrm{~d} \widehat{z}}\right)
$$

where the second equality in Eq. (7) follows from our choice of $\widehat{L}$, and $\hat{f}$ is the source frequency in hertz.

In a typical example of mesoscale eddies discussed by $\mathrm{WU}_{\mathrm{U}}\left(\mathrm{Wu}\right.$ et al., 2001), $\left(\frac{\mathrm{d} \widehat{u}_{0}}{\mathrm{~d} z}\right)=0.002 \mathrm{~s}^{-1}$ with a surface current speed of $\widehat{U}_{0}=1.0 \mathrm{~m} / \mathrm{s}$. It follows that:

$$
\xi \cong \frac{0.5}{f}
$$

Consequently, $\xi$ is considerably small even at lower acoustic frequencies. For instance, when $\widehat{f}=$ $50 \mathrm{~Hz}$, we have $\xi \leq O\left(M^{\frac{1}{2}}\right)$.

Then Eq. (5) becomes

$$
\begin{aligned}
\mu \rho_{0} \nabla \cdot\left(\frac{1}{\rho_{0}} \nabla p_{1 t}\right)= & 2 M \mu n^{2} u_{0} p_{1 x t t} \\
& -2\left[M \xi /\left(\mu \rho_{0}^{2}\right)\right] G p_{1 z x} \\
& +\mu^{2} n^{2} p_{1 t t}
\end{aligned}
$$

Consequently, terms of $O(M \xi)$ will be negligibly small compared to $M$ (ROBERTSON et al., 1985), then Eq. (9) reduces to

$$
\mu \rho_{0} \nabla \cdot\left(\frac{1}{\rho_{0}} \nabla p_{1}\right)=2 M \mu n^{2} u_{0} p_{1 x t}+\mu^{2} n^{2} p_{1 t}
$$

Then Eq. (10) may be written in dimensional form as follows

$$
\widehat{c}_{\mathrm{w}}^{2} \widehat{\rho}_{0} \nabla \cdot\left(\frac{1}{\widehat{\rho}_{0}} \nabla \widehat{p}_{1}\right)=2 \widehat{u}_{0} \frac{\partial^{2} \widehat{p}_{1}}{\partial \widehat{t} \partial \hat{x}}+\frac{\partial^{2} \widehat{p}_{1}}{\partial \widehat{t}^{2}}
$$


The first term on the right hand of Eq. (11) is due to current effect of eddies.

For the homogeneous media below water, the pressure $\hat{p}_{\mathrm{h}}$ satisfies the governing equations:

$$
\widehat{c}_{\mathrm{h}}^{2} \nabla^{2} \widehat{p}_{\mathrm{h}}=\frac{\partial^{2} \widehat{p}_{\mathrm{h}}}{\partial \hat{t}^{2}}
$$

where $\widehat{c}_{\mathrm{h}}$ is the sound speed in each layer.

\subsection{Numerical Algorithm}

In the following, we describe briefly the propagation algorithm for the equations mentioned above, which consists of a finite-difference scheme implemented on a uniform grid with the same size $\Delta$ in the $x$ - and $z$-axis. Then the classical explicit second-order finite-difference scheme for Eq. (11) yields

$$
\begin{aligned}
& \widehat{c}_{\mathrm{w}}^{2} \hat{\rho}_{0} \hat{\nabla} \cdot\left(\frac{1}{\hat{\rho}_{0}} \hat{\nabla} \hat{p}_{1}\right)=\widehat{c}_{\mathrm{w}, i j}^{2} \hat{\rho}_{0, i j} \\
& \times\left\{\frac{1}{\hat{\rho}_{0, i j}} \frac{\widehat{p}_{1, i+1, j}-2 \widehat{p}_{1, i j}+\widehat{p}_{1, i-1, j}}{\Delta x^{2}}\right. \\
& -\frac{1}{\widehat{\rho}_{0 . i j}} \frac{\widehat{p}_{1, i, j+1}-2 \widehat{p}_{1, i j}+\widehat{p}_{1, i, j-1}}{\Delta z^{2}} \\
& +\frac{1}{4 \Delta x^{2}}\left(\frac{1}{\hat{\rho}_{0, i+1, j}}-\frac{1}{\hat{\rho}_{0, i-1, j}}\right) \cdot\left(\hat{p}_{1, i+1, j}-\widehat{p}_{1, i-1, j}\right) \\
& \left.+\frac{1}{4 \Delta z^{2}}\left(\frac{1}{\hat{\rho}_{0, i, j+1}}-\frac{1}{\hat{\rho}_{0, i, j-1}}\right) \cdot\left(\hat{p}_{1, i, j+1}-\hat{p}_{1, i, j-1}\right)\right\}
\end{aligned}
$$

$$
\begin{gathered}
\hat{p}_{1, t t}=\frac{\widehat{p}_{1, i j}^{n+1}-2 \widehat{p}_{1, i j}^{n}+\widehat{p}_{1, i j}^{n-1}}{\Delta t^{2}} \\
\widehat{p}_{1, x t}=\frac{\hat{p}_{1, i+1, j}^{n}-\widehat{p}_{1, i+1, j}^{n-1}-\widehat{p}_{1, i-1, j}^{n}+\widehat{p}_{1, i-1, j}^{n-1}}{2 \Delta \widehat{x} \Delta \widehat{t}}
\end{gathered}
$$

The first-order PML introduced by Kormann are used in this paper. It is worthwhile to emphasize that, to observe the reflections yielded by the eddy clearly, spurious numerical reflections at the lateral PML boundaries must be at least one order smaller than the magnitude of the physical phenomena of interest.

\section{Numerical Results}

In this section we investigate the effect of eddies on seismic wave propagation using the algorithm described in Sect. 2.

\subsection{Forward Modeling}

Firstly, we extract the center region of the eddy to observe seismic wave propagation. Figure 2a shows a sketch of the numerical model. The source with dominant frequency $40 \mathrm{~Hz}$ is place at mid-range, $50 \mathrm{~m}$ deep. The space and time discretization are $\Delta x=\Delta z=1$ and $\Delta t=1.25 \times 10^{-4} \mathrm{~s}$, respectively. The region we simulated is $4,000 \mathrm{~m}$ long and 2,600 $\mathrm{m}$ deep and the PML thickness is set to four wavelengths of the Ricker wavelet. The depth of the water is $2,200 \mathrm{~m}$, and the properties of each layer
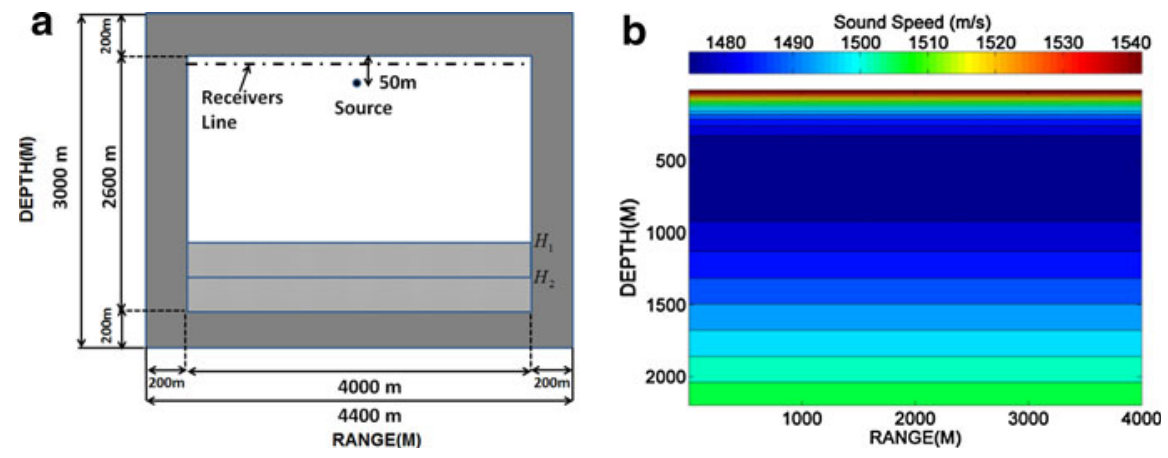

Figure 2

a Sketch of the numerical model with the positions of the source and the receivers; $\mathbf{b}$ sound speed map of water around the center of the eddy 
below water are listed in Table 1 . There are two reflectors $\left(H_{1}, H_{2}\right)$ at the depth of 2,200 and 2,400 m, respectively. In the following, we will investigate the characteristic of seismic wave propagation for both having eddy case and no-eddy case. For having eddy case, sound speed map of water around the center of the eddy is illustrated in Fig. 2b. We can see that sound speed in the upper part of the eddy vary more quickly than that in the lower part if the eddy. And in the center of the eddy, sound speed has little change.

Figure 3 illustrates the wavefields at time $1.592 \mathrm{~s}$ both in region containing the eddy and in the same region where the perturbation of the eddy is absent. From the plots, we can see that the algorithm

\section{Table 1}

Properties of the layers below water in Fig. $2 a$

\begin{tabular}{llll}
\hline Layers & P wave speeds $(\mathrm{m} / \mathrm{s})$ & Density $\left(\mathrm{g} / \mathrm{cm}^{3}\right)$ & Thickness $(\mathrm{m})$ \\
\hline 1 & 2,000 & 2.1 & 200 \\
2 & 4,000 & 2.5 & 200 \\
\hline
\end{tabular}

The layers are numbered from the sea floor. described in this paper gives no visible numerical dispersion. At the resolution of the figure, no reflected waves can be seen coming from the PML domain. When the wave travels through the eddy, the inhomogeneous velocities of the wavefields cause weak reflected waves (Fig. 3a). To perform a further comparison between having eddy and no-eddy case, the wavefront curves in the zoom between 2,000 and 2,600 $\mathrm{m}$ are plotted in Fig. 3c. We can see that the two curves are distinguished and in layers below water the difference are larger than that in water.

Now we further examine the synthetic seismograms generated from the numerical model with the eddy. To observe the reflections from the eddy clearly, a zoom of the synthetic seismograms between 0 and $2.9 \mathrm{~s}$ are depicted in Fig. 4b. We can see some weak reflected waves in the upper and lower part of Fig. 4b. The core of the eddy shows low reflectivity, suggesting homogeneous water mass. Moreover, the amplitude of these weak reflected waves is of order $10^{-4}$ while the amplitude of the reflections from the reflectors below water is of order $10^{-2}$.

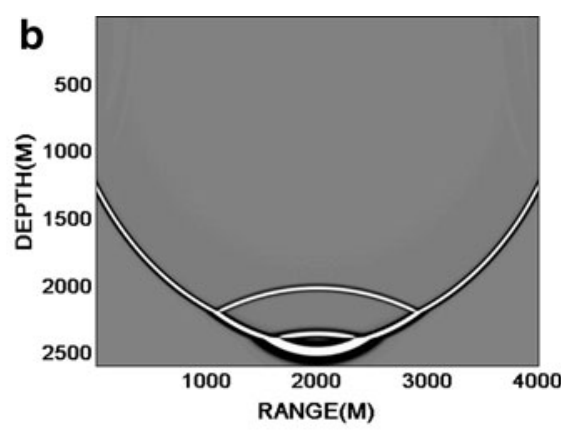

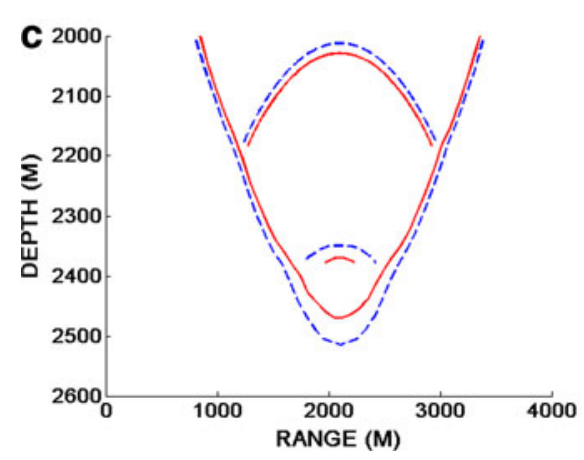

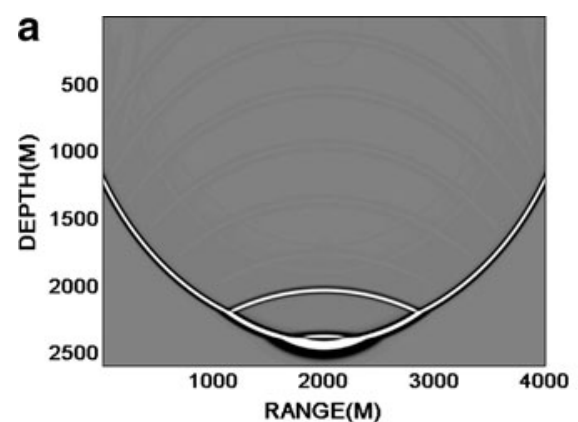

Figure 3

a Wavefields at time $1.592 \mathrm{~s}$ computed with Eq. (11) for having eddy case. b Wavefields at time $1.592 \mathrm{~s}$ for no-eddy case. c Comparison of wavefront between having eddy (solid line) and no-eddy case (dashed line) 

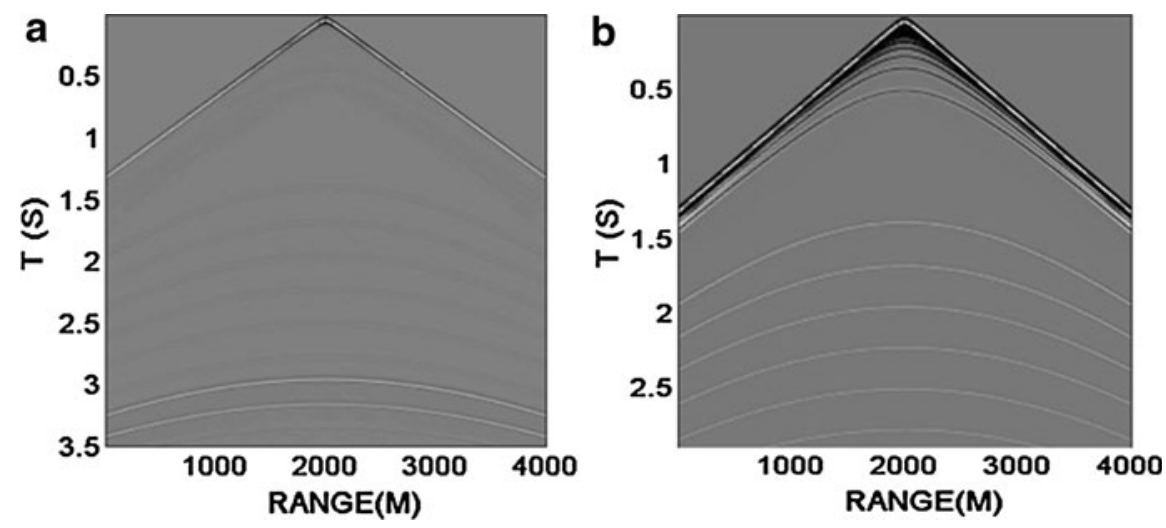

Figure 4

Synthetic seismograms for the numerical model with the eddy (a) and a zoom between $t=0 \mathrm{~s}$ to $2.9 \mathrm{~s}$ (b)
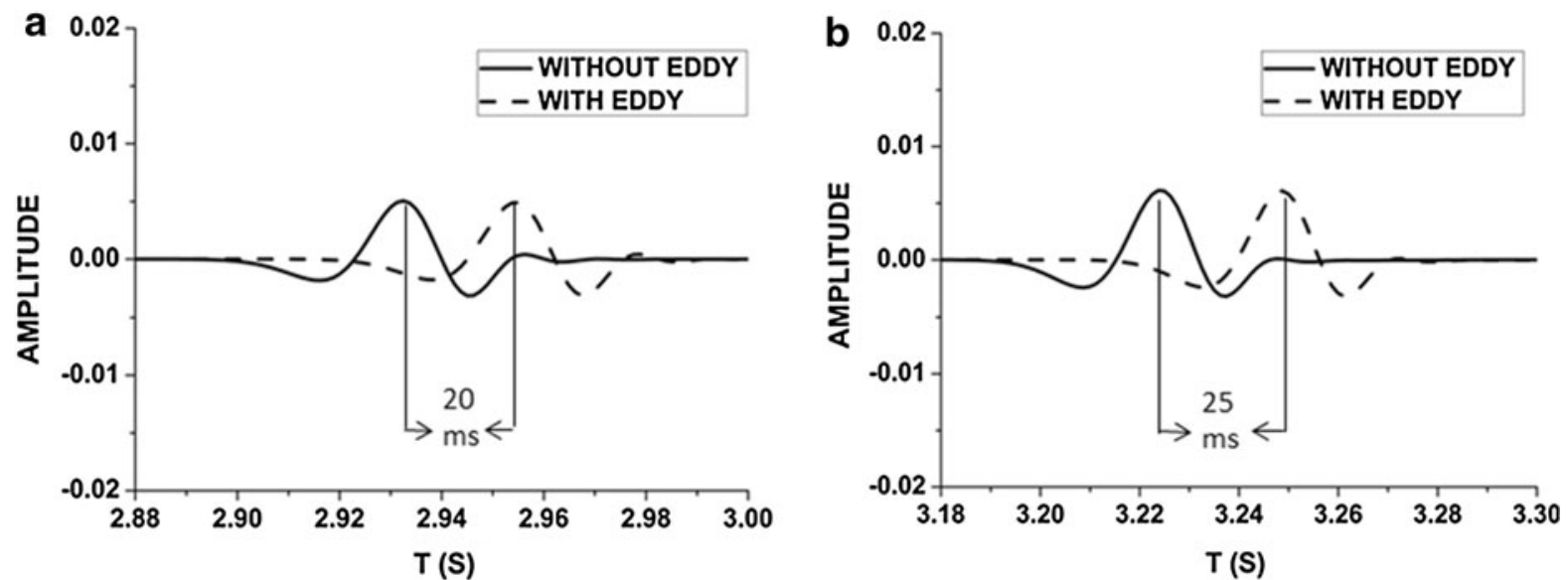

Figure 5

Zoom on the recorded signals at two ranges along the receiver line for having eddy (dashed line) and no-eddy case (solid line): a 2,000 $\mathrm{m}$ and b $4,000 \mathrm{~m}$

To investigate the effect of the eddy on the reflections from layers lying below water, we compare the waveforms generated by the first reflector $H_{1}$ between having eddy and no-eddy case. Figure 5 shows the signal recorded at two ranges. We can see that seismic wave propagates more slowly because of the perturbation of the eddy. For the numerical model of Fig. 2, the time difference increases from $20 \mathrm{~ms}$ at offset of $0 \mathrm{~m}$ (Fig. 5a) to $25 \mathrm{~ms}$ at offset of 2,000 m (Fig. 5b).

\subsection{Pre-Stack Depth Migration}

In this section we will use several more general models to investigate the effect of the eddy on seismic imaging. Three models (Fig. 6) are considered: two five-layer models (labeled Model 1 and Model 2) and a three-layer model (labeled Model 3). The first layer of each model is water with eddy and its velocity is plotted in Fig. 2b. In Models 1 and 2, there are four flat layers with the same thickness below water. In Model 3, the two layers below water are separated by a dipping interface. Here we used the algorithm described in Sect. 2 to obtain the seismic data and then applied pre-stack reverse time depth migration to them. For each model, the shot spacing is $10 \mathrm{~m}$, and the receiver spacing is $10 \mathrm{~m}$. It is necessary to point out that, during depth migration, the migration velocity we used in water is the velocity without eddy. Moreover, to remove the 

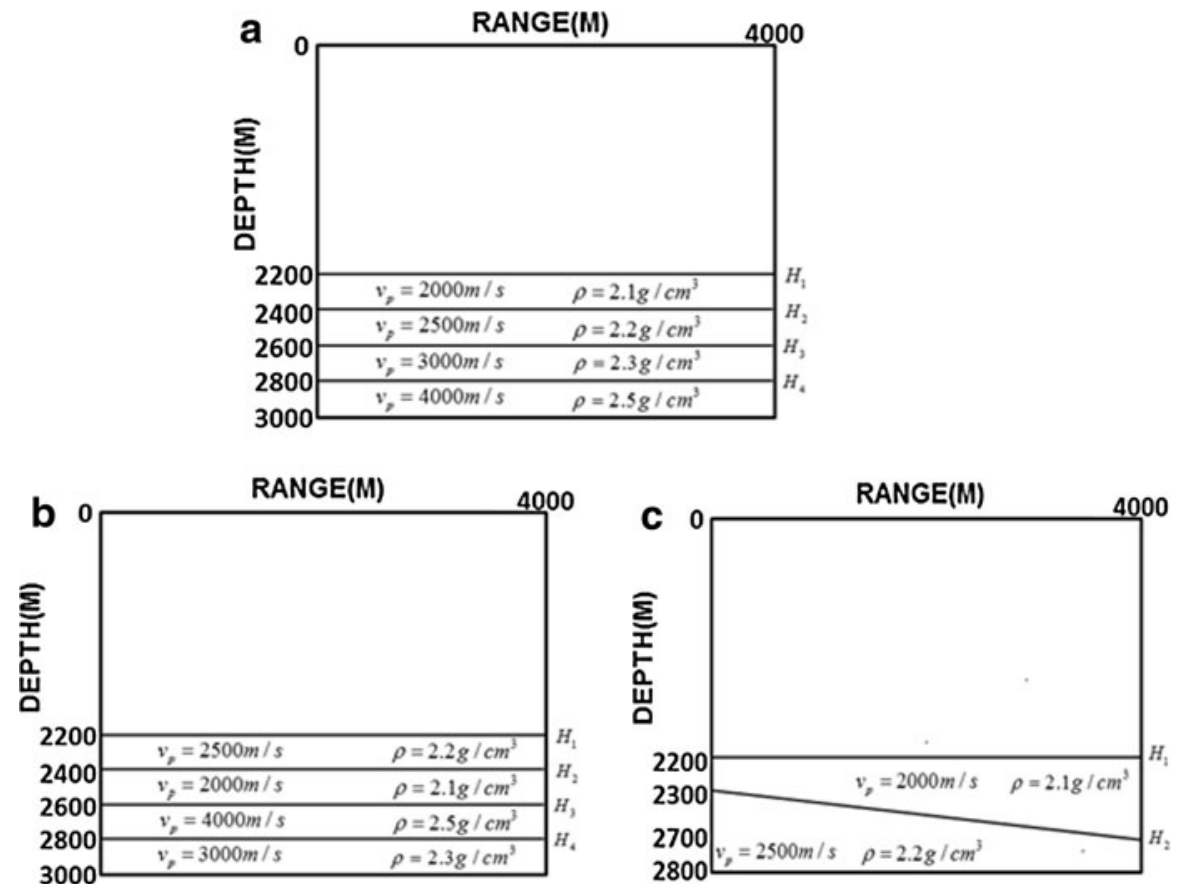

Figure 6

Model 1 (a); Model 2 (b); Model 3 (c)

interference factors produced by velocity inaccuracy in layers below water, the migration velocity in these layers is exactly the real velocity.

Figure $7 \mathrm{a}, \mathrm{b}$ show the images migrated with Model 1 for no-eddy and having eddy case. Comparing these two images, one can observe that the four reflectors in Fig. $7 \mathrm{~b}$ are positioned incorrectly due to the perturbation of the eddy. Further, the depth perturbation of reflectors increases with the migration velocity of the layer. For instance, the depth of the first reflector is $2,220 \mathrm{~m}$, while the depth of the forth reflector increases to $2,840 \mathrm{~m}$.

Table 2 lists the depth perturbation of reflectors for the three models, where $\Delta d$ is the depth perturbation. Thus, we can conclude that the image perturbation of reflectors introduced by the eddy is proportion to the migration velocities. The proportional coefficient is 0.01 , which is half the travel time difference between having eddy and no-eddy case at the zero offset trace. As a consequence, the relationship between depth perturbation of reflectors and the migration velocity below the reflectors can be summarized as following:

$$
\Delta d=v_{\mathrm{m}} \times \Delta t / 2
$$

where $\Delta d=d_{1}-d_{2}, \Delta t=t_{1}-t_{2}, d_{1}\left(d_{2}\right)$ is the depth of the reflectors for having eddy (no-eddy case). $t_{1}\left(t_{2}\right)$ is the traveltime of reflections from the reflectors for having eddy (no-eddy case) at zero offset. $v_{\mathrm{m}}$ is migration velocity below the reflector.

Next we consider a complex model (Fig. 8a) with constant density to test the formulation (Eq. 16). As shown in Fig. 8b, the model consists of four complicated layers below water. Figure 8c is the image migrated with seismic data computed with the algorithm described in this paper while the dashed lines are the true positions of the reflectors. We found that the formulation (Eq. 16) is valid under the assumption of homogeneous media below water, small offset angle $\left(<10^{\circ}\right)$ and smallcurvature reflectors.

\section{Discussion and Conclusions}

We have discussed the perturbation of the eddy around its center. However, as mentioned in the 

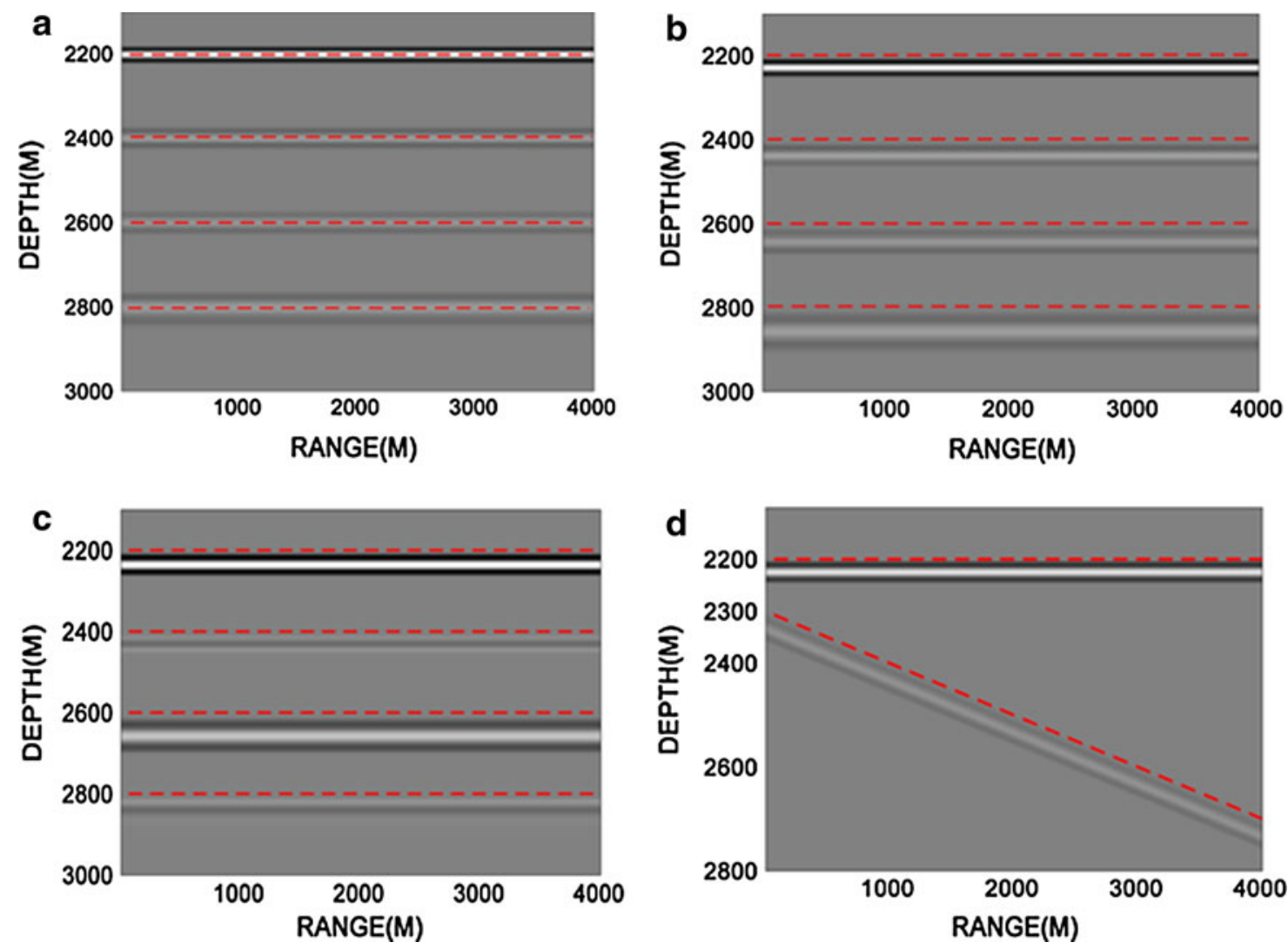

Figure 7

Images migrated with Model 1 for no-eddy case (a); Model 1 for having eddy case (b); Model 2 (c); Model 3 (d). The dashed lines represent the true position of the reflections

Table 2

Depth perturbation of reflectors in three models

\begin{tabular}{llll}
\hline Reflectors & \multicolumn{2}{l}{$\Delta d(\mathrm{~m})$} & \\
\cline { 2 - 4 } & Model 1 & Model 2 & Model 3 \\
\hline$H_{1}$ & 20 & 25 & 20 \\
$H_{2}$ & 25 & 20 & 25 \\
$H_{3}$ & 30 & 40 & - \\
$H_{4}$ & 40 & 30 & - \\
\hline
\end{tabular}

beginning of the paper, the eddy is moving and its effective radius is $150 \mathrm{~km}$ in horizontal range, so in deepwater field development we should investigate the effect of the eddy on seismic wave propagation and seismic imaging throughout the area of its influence.

As shown in Fig. 1, the region we simulated is $300 \mathrm{~km}$ long and 2,600 $\mathrm{m}$ deep. In addition, we assume that the material properties of two layers below water are the same as listed in Table 1. The zero-offset seismic profiling throughout the eddy is described in Fig. 9a. The reflections from the eddy is weak, and it can be checked more clearly in the zoom between 0.1 and $2.9 \mathrm{~s}$ (Fig. 9b). In Fig. 9b highly reflective lens-like structures can be observed, and this result coincides with the observations in seismic oceanography (Dong et al., 2010; Song et al., 2008). The core shows low reflectivity. Moreover, the reflections in the upper part of the eddy are steeplydipping while those in the lower part are mainly horizontal. These complex reflections in the eddy can perturbate the primary reflections from sedimentary reflectors.

Figure 10 shows the details of the reflections from the first reflector $H_{1}$ for having eddy and no-eddy case. It can be seen that the arrival time in Fig. 10a is slower than that in Fig. 10b. The difference of arrival 

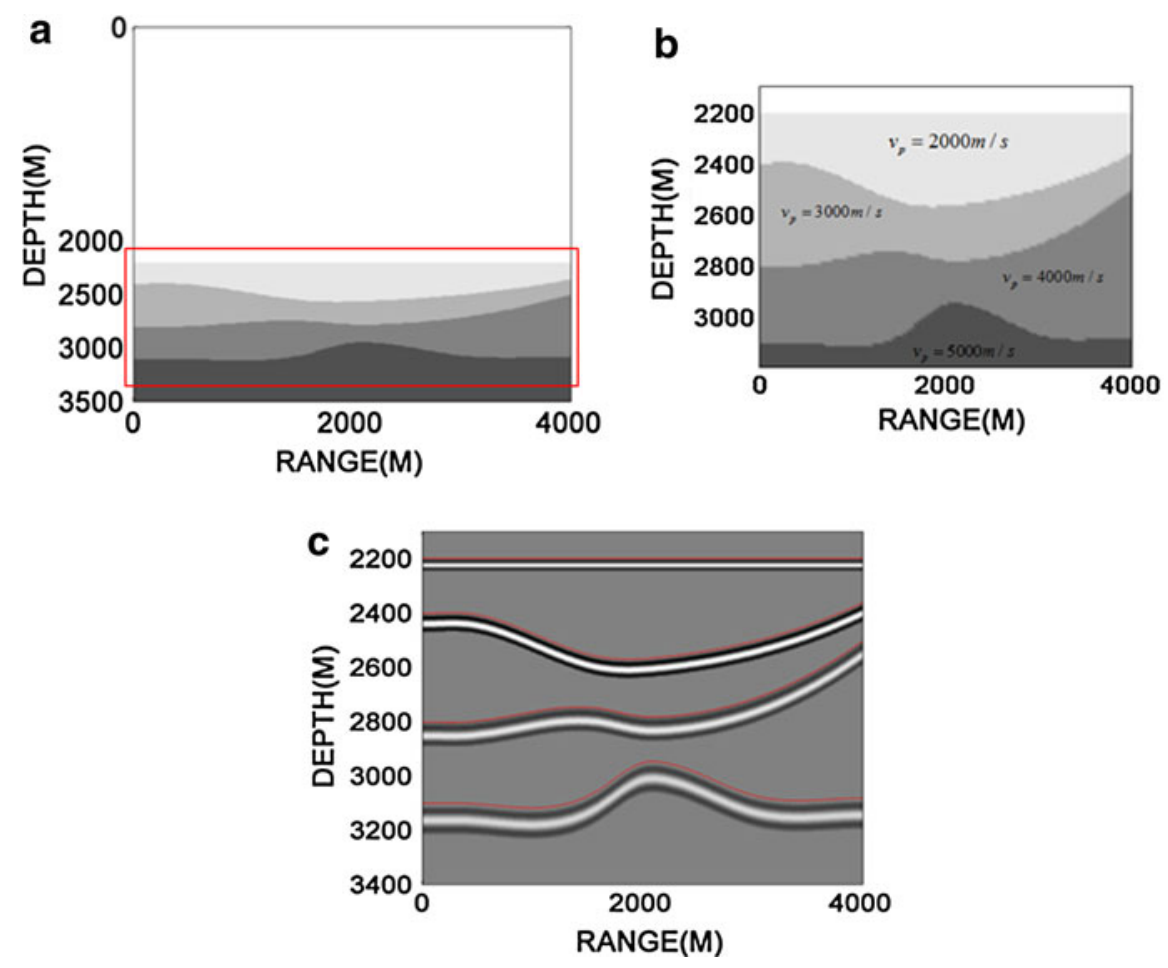

Figure 8

The complex model (a) and the detail of layers below water (b); images migrated with the complex model (c)
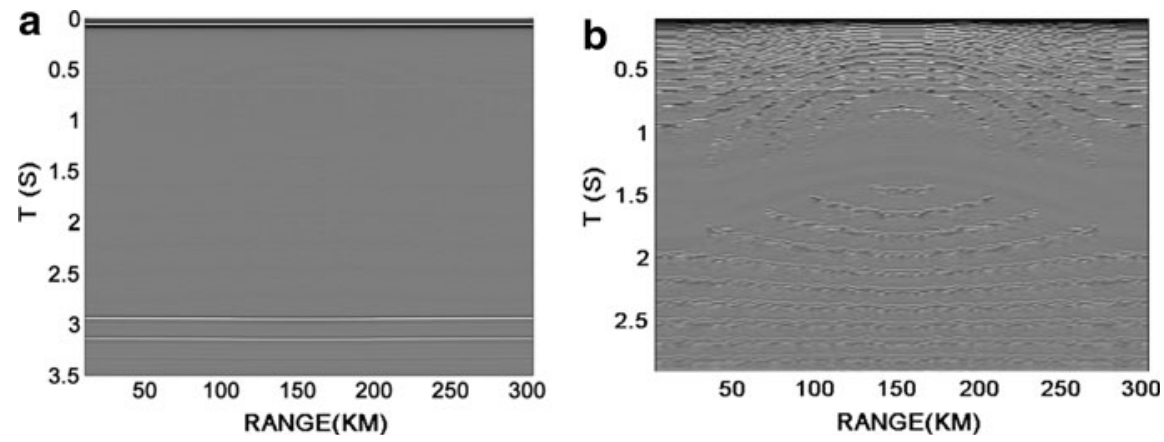

Figure 9

a Zero-offset seismic profiling throughout the eddy. b The zoom of zero-offset seismic profiling between 0.1 and $2.9 \mathrm{~s}$

time increases from $10.5 \mathrm{~ms}$ at the edge of the eddy to $20 \mathrm{~ms}$ at the center of the eddy. Further, the arrival-time difference is symmetric, as expected. As mentioned above, the traveltime difference at zerooffset trace is important, so Fig. 11 gives the description of $\Delta t$ throughout the eddy.

Then we can use Eq. (16) and Fig. 11 to estimate the image perturbation of reflectors caused by the eddy. We obtain that the depth of the first reflector $H_{1}$ increases from $2,210 \mathrm{~m}$ at the edge of the eddy to $2,220 \mathrm{~m}$ at the center of the eddy while the depth of the second reflector $\mathrm{H}_{2}$ increases from 2,420 to $2,440 \mathrm{~m}$. Thus, the structure of the flat reflector becomes distorted because of the perturbation of the eddy.

The purpose of this paper is to study the effect of eddies on seismic wave propagation and seismic imaging. To highlights the inhomogeneous effects of 

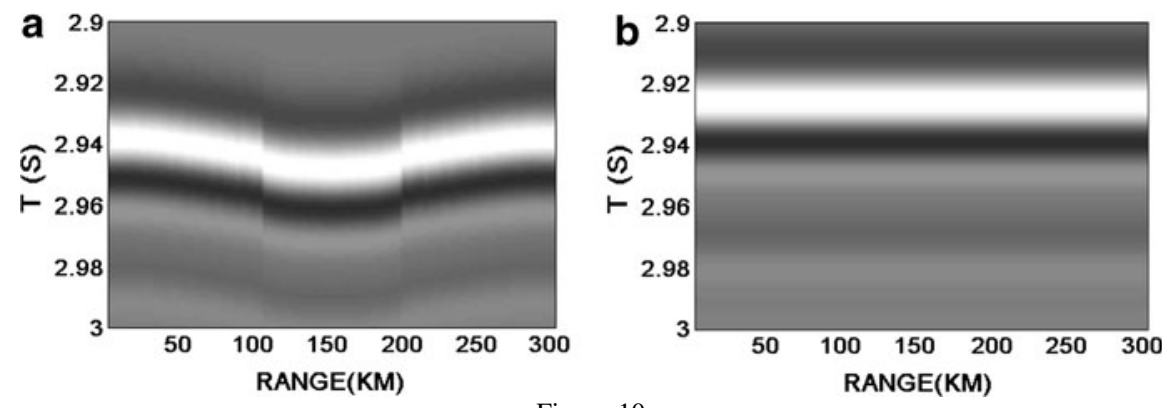

Figure 10

a Zoom of zero-offset seismic profiling between 2.9 and $3.0 \mathrm{~s}$ for having eddy. b Zoom of zero-offset seismic profiling between 2.9 and $3.0 \mathrm{~s}$ for no-eddy case

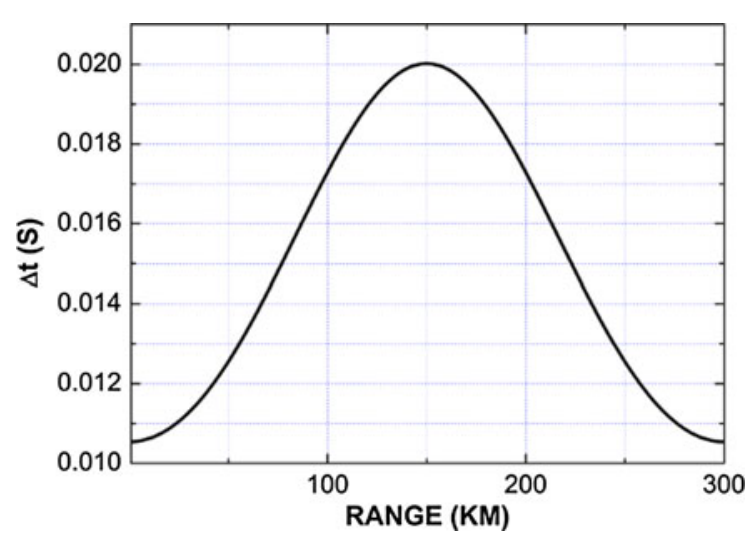

Figure 11

$\Delta t$ throughout the eddy

water, we derive an appropriate partial differential equation for the pressure field in water with eddies, including current effects. Based on numerical calculations, we finally obtain that:

1. There are weak reflections in the eddy, the amplitude of these weak reflected waves is of order $10^{-4}$, and is two orders smaller than that from layers below water.

2. Because of the perturbation of the eddy, seismic wave propagates more slowly. This leads to incorrect estimation of the depths of reflectors. A simple formulation is given to estimate this image perturbation.

3. As approaching the center of the eddy, the effect of the eddy on seismic imaging becomes larger. Thus the migrated images of flat reflectors below the eddy become distorted.
In sum, the eddy plays an important role in seismic wave propagation and seismic imaging, and it can't be neglected in deepwater field development.

\section{Acknowledgments}

The financial support from the National Natural Science Foundation of China (Grant No. 41074097) and the "973" programs (No. 2009CB219405-1) are gratefully acknowledge.

\section{Appendix}

In this appendix, we will derive Eq. (5) using asymptotic method.

Substituting Eqs. (2)-(4f) into Eq. (1a) provides

$$
\begin{aligned}
& \delta \widehat{L} \widehat{c}_{0}^{-1} T^{-1} \frac{\partial \rho_{1}}{\partial t} \\
& \quad+\nabla\left(M \rho_{0} \mathbf{v}_{0}+\delta \rho_{0} \mathbf{v}_{1}+M \delta \rho_{1} \mathbf{v}_{0}+\delta^{2} \rho_{1} \mathbf{v}_{1}\right) \\
& \quad=0
\end{aligned}
$$

where $M$ denotes the Mach number $\widehat{U}_{0} / \widehat{c}_{0}$.

When $\delta=0$, Eq. (17) is identically zero since $\widehat{\mathbf{v}}_{0}$ depends only on $z$ and has no vertical component. Keeping terms of $O(\delta)$, Eq. (17) reduces to

$$
\mu \frac{\partial \rho_{1}}{\partial t}+\nabla \cdot\left(\rho_{0} \mathbf{v}_{1}+M \rho_{1} \mathbf{v}_{0}\right)=0
$$

where

$$
\mu=\widehat{L} \widehat{c}_{0}^{-1} \widehat{T}^{-1}
$$


Since we anticipate that the parameter $\mu$ is order unity for acoustic waves, Eq. (18) represents a scaled conservation of mass equation.

Now we consider the scaling of Eq. (1b). Substituting Eqs. (4a)-(4f) into Eq. (1b) and algebraically simplifying, it yields

$$
\begin{aligned}
& \left(\rho_{0}+\delta \rho_{1}\right)\left[\mu \delta \frac{\partial \mathbf{v}_{1}}{\partial t}+M^{2}\left(\vec{v}_{0} \cdot \nabla\right) \mathbf{v}_{0}+M \delta\left(\vec{v}_{0} \cdot \nabla\right) \mathbf{v}_{1}\right. \\
& \left.+M \delta\left(\mathbf{v}_{1} \cdot \nabla\right) \mathbf{v}_{0}+\delta^{2}\left(\mathbf{v}_{1} \cdot \nabla\right) \mathbf{v}_{1}\right]=-\nabla\left(M^{2} p_{0}+\mu \delta p_{1}\right)
\end{aligned}
$$

Further, $\delta=0$ and the left side of Eq. (20) is identically zero since $\mathbf{v}_{0}$ depends only on $z$ and has no vertical component. Eliminating ambient terms from the linearized version of Eq. (20) provides

$$
\mu \frac{\partial \mathbf{v}_{1}}{\partial t}+M\left(\mathbf{v}_{0} \cdot \nabla\right) \mathbf{v}_{1}+M\left(\mathbf{v}_{1} \cdot \nabla\right) \mathbf{v}_{0}=-\frac{\mu}{\rho_{0}} \nabla p_{1}
$$

Finally, we scale Eq. (1c) in the same manner, using $n=\widehat{c}_{0} / \widehat{c}_{w}$ as the index of refraction. With the scaled variables already defined, Eq. (1c) becomes

$$
\begin{aligned}
& n^{-2}\left(\mu \delta \frac{\partial \rho_{1}}{\partial t}+\delta \mathbf{v}_{1} \cdot \nabla \rho_{0}+\delta M \mathbf{v}_{0} \cdot \nabla \rho_{1}+\delta^{2} \mathbf{v}_{1} \cdot \nabla \rho_{1}\right) \\
& =M^{3} \mathbf{v}_{0} \cdot \nabla p_{0}+\mu M \delta \mathbf{v}_{0} \cdot \nabla p_{1}+M^{2} \delta \mathbf{v}_{1} \cdot \nabla p_{0} \\
& \quad+\mu \delta^{2} \mathbf{v}_{1} \cdot \nabla p_{1}+\mu^{2} \delta \frac{\partial p_{1}}{\partial t}
\end{aligned}
$$

when $\delta=0$, the reduced equation is the balance condition to be satisfied identically by the ambient terms. Under the condition $\delta=O\left(M^{2}\right)$, where, as usual, $M \ll 1$, the appropriate simplification of Eq. (1c) is

$$
\begin{aligned}
& n^{-2}\left(\mu \frac{\partial \rho_{1}}{\partial t}+\mathbf{v}_{1} \cdot \nabla \rho_{0}+M \mathbf{v}_{0} \cdot \nabla \rho_{1}\right) \\
& \quad=\mu M \mathbf{v}_{0} \cdot \nabla p_{1}+\mu^{2} \frac{\partial p_{1}}{\partial t}
\end{aligned}
$$

That this condition on $\delta$ is reasonable follows from Eq. (4b), which implies that

$$
\delta=O\left(\left|\widehat{\mathbf{v}}_{\mathrm{w} 1}\right| / \widehat{c}_{0}\right)
$$

Since $M$ in the ocean is never bigger than $10^{-3}$, the condition $\delta=O\left(M^{2}\right)$ and Eq. (24) imply that the magnitude of the velocity induced by the acoustic disturbance is no more than about $10^{-3} \mathrm{~ms}^{-1}$. This is a reasonable and conservation means the acoustically induced disturbance is smaller than the ambient flow by a scaling factor of order Mach number.

Next, we wish to extract from Eqs. (18), (21) and (23) a single equation for acoustic pressure. It is a straightforward calculation to show that these equations reduce to the pair

$$
\begin{aligned}
\mu \rho_{0} \nabla \cdot\left(\frac{1}{\rho_{0}} \nabla p_{1}\right)= & 2 M \mu n^{2} u_{0} P_{1 x t} \\
& -2\left[M /\left(\mu \rho_{0}\right)\right] u_{0}^{\prime} w_{x}+\mu^{2} n^{2} p_{1 t t} \\
-p_{1 z}= & \rho_{0} \frac{\partial w}{\partial t}+(M / \mu) \rho_{0} u_{0} w_{x}
\end{aligned}
$$

where $u_{0}^{\prime}=d u_{0} / d z$, and $w=\mathbf{v}_{1} \cdot \mathbf{k}$ is the $z$ component of acoustic velocity $\mathbf{v}_{1}$. The dimensionless ambient current component $u_{0}(z)$ is defined in Eq. (4a). Now, by taking the $t$ derivative of Eq. (25a) and the $x$ derivative of Eq. (25b), the $w$ can be eliminated to give the following third-order equation, correct to terms of order $M$ :

$$
\begin{aligned}
\mu \rho_{0} \nabla \cdot\left(\frac{1}{\rho_{0}} \nabla p_{1 t}\right)= & 2 M \mu n^{2} u_{0} p_{1 x t t} \\
& -2\left[M /\left(\mu \rho_{0}^{2}\right)\right] u_{0}^{\prime} p_{1 z x} \\
& +\mu^{2} n^{2} p_{1 t t}
\end{aligned}
$$

Picking $\widehat{T}=(2 \pi \widehat{f})^{-1}$ ensures that time derivatives are $O(1)$, where $\hat{f}$ is the frequency of the source in hertz. Also, we choose the reference wavenumber $\hat{k}_{0}\left(\hat{k}_{0}=2 \pi \hat{f} / \widehat{c}_{0}\right)$ as our length scale, and then we have $\widehat{L}=\widehat{c}_{0} \widehat{T}$. Consequently, $\mu=1$.

\section{REFERENCES}

BAER R.N. (1980), Calculations of sound propagation through an $e d d y$, Journal Acoust. Soc. Am. 67, 1180-1185.

Benitez-Nelson C.R., McGillicuddy D.J. (2008), Mesoscale Physical Biological Biogeochemical Linkages in the Open Ocean: Results from the EFLUX and EDDIES Programs, Deep Sea Research Part II. 55, 1133-1138.

Biescas B., Sallare S.V., Pelegr J.L., et al. (2008), Imaging meddy fine structure using multichannel seismic reflection data, Geophysical Research Letters. 35, L11609, doi:10.1029/2008 GL033971.

Dong C.Z., Song H.B., BAI Y., et al. (2010), The latest development of seismic oceanography, Progress in Geophys (in Chinese). 25, 109-123. 
Gonella J., Michon D. (1988), Deep internal waves measured by seismic reflection with in the eastern Atlantic water mass (in French with English abstract), Comptes Renduse Academie Des Sciences (SeriesIIA). 306, 781-787.

Henrick R.F., Jacobson M.J., Siegmann W.L. (1980), General effects of currents and sound speed variations on short range acoustic transmission in cyclonic eddies, Journal Acoust. Soc. Am. 67, 121-134.

Henrick R.F., Siegmann W.L., Jacobson M.J. (1977), General analysis of ocean eddy effects for sound transmission applications, Journal Acoust. Soc. Am. 62, 860-870.

Holbrook W.S., Paramo P., Pearse S., et al. (2003), Thermohaline fine structure in an oceanographic front from seismic reflection profiling, Science. 301, 821-824.

Hough G., Green J., Fish P., et al. (2011), A geomorphological mapping approach for the assessment of seabed geohazards and risk, Mar Geophys Res. 32, 151-162.

Hu Y., Liu H.S., CHEN J., Xu J. (2009), Recent progress in seismic oceanography, Advance in Earth Science. 24, 1094-1104.

Jensen F.B., Kuperman W.A., Porter M.B., et al. Computational Ocean Acoustics, (Springer, New York 2000).

JiAn Y.J., Zhang J., Liu Q.S., Wang Y.F. (2009), Effect of mesoscale eddies on underwater sound propagation, Applied Acoustics. 70, 432-440.

Kormann J., Сово P., Biescas B., et al. (2010), Synthetic modelling of acoustical propagation applied to seismic oceanography experiments, Geophysical Research Letters. 37, L00D90, doi: 10.1029/2009GL041763.

Kormann J., Совo P., Prieto A. (2008), Perfectly matched layers for modelling seismic oceanography experiments, Journal of Sound and Vibration. 317, 354-365.

Kormann, J., Cobo P., Recuero M., Biescas B., Sallarés V. (2009), Modelling seismic oceanography experiments by using first- and second- order complex frequency shifted perfectly matched layers, Acta Acust. Acust. 95, 1104-1111, doi:10.3813/ AAA. 918242 .
Krahmann G., Brandt P., Klaeschen D., Reston T. (2008), Middepth internal wave energy off the Iberian Peninsula estimated from seismic reflection data, J. Geophys. Res. 113, C12016, doi: 10.1029/2007JC00467.

LiU Q.H., TAO J. (1997), The perfectly matched layer for acoustics waves in absorptive media, Journal Acoust. Soc. Am. 102, 2072-2084.

Nysen P.A., Power P.S. (1978), Sound propagation through an East Australian Current eddy, Journal Acoust. Soc. Am. 63, 1381-1388.

Pinheiro L.M., Song H., Ruddick B., Dubert J. (2010), Detailed 2-D imaging of the Mediterranean outflow and meddies off $W$ Iberia from multichannel seismic data, Journal of Marine Systems, 79, 89-100.

Robertson J.S., Siegmann W.L., Jacobson M.J. (1985), Current and current shear effects in the parabolic approximation for underwater sound channels, Journal Acoust. Soc. Am. 77, 1768-1780.

Song H.B., Dong C.Z., Chen L., Song Y. (2008), Reflection seismic methods for studying physical oceanography: Introduction of seismic oceanography, Progress in Geophys (in Chinese). 23, 1156-1164.

Song H.B., Luis P., Wang D.X., et al. (2009), Seismic images of ocean meso-scale eddies and internal waves, Chinese Journal Of Geophysics. 52, 2775-2780.

Temkin S., Elements of Acoustics, (Wiley, New York 1981).

VARTANYAN G.S. (2010), Regional geodynamic monitoring system for ensuring safety in geological and exploratory production of oil and gas, Atmospheric and Oceanic Physics. 46, 952-964.

Wood W.T., Holbrook W.S., Sen M.K., et al. (2008), Full wave form inversion of reflection seismic data for ocean temperature profiles, Geophysical Research Letters. 35, L04608, doi: 10.1029/2007 GL032359.

Wu P.M., Guo X.G., Wu R.S. (2001), Analyses of sound velocity field in southwest sea area off Hengchun of Taiwan, J Oceanogr Taiwan Strait (in Chinese). 20, 279-286. 